\title{
Entre ombres et lumières, le parcours singulier d'une féministe pacifiste, Jeanne Mélin (1877-1964)
}

Isabelle VAHÉ

\section{(2) OpenEdition}

1 Journals

Édition électronique

URL : https://journals.openedition.org/clio/4502

DOI : $10.4000 /$ clio.4502

ISSN : 1777-5299

Éditeur

Belin

Édition imprimée

Date de publication : 1 novembre 2006

ISBN : 2-85816-867-9

ISSN : $1252-7017$

Référence électronique

Isabelle VAHÉ, «Entre ombres et lumières, le parcours singulier d'une féministe pacifiste, Jeanne Mélin (1877-1964) », Clio. Histoire, femmes et sociétés [En ligne], 24 | 2006, mis en ligne le 23 août 2013, consulté le 22 avril 2022. URL : http://journals.openedition.org/clio/4502 ; DOI : https://doi.org/ $10.4000 /$ clio.4502

Ce document a été généré automatiquement le 22 avril 2022

Tous droits réservés 


\title{
Entre ombres et lumières, le parcours singulier d'une féministe pacifiste, Jeanne Mélin (1877-1964)
}

\author{
Isabelle VAHÉ
}

1 Souhaitant travailler sur les journaux intimes de femmes, j'ai découvert à la Bibliothèque Historique de la Ville de Paris celui de Jeanne Mélin (1877-1964), une figure méconnue des mouvements féministes et pacifistes français ${ }^{1}$. Actrice, selon la belle expression de Françoise Thébaud ${ }^{2}$, d'un « formidable bouleversement entre les sexes ", Jeanne Mélin traverse et vit, quelquefois douloureusement, les différentes étapes de l'histoire de ces deux mouvements de la fin du XIX ${ }^{e}$ siècle, qui en voit l'émergence, jusqu'aux années 1960.

2 Pacifiste, féministe, écrivaine, propagandiste, Jeanne Mélin parcourt l'Europe et rencontre plusieurs prix Nobel. Elle milite inlassablement pour la paix et le droit de vote des femmes. Elle est aussi une participante active de l'histoire géopolitique de ce siècle : la Grande Guerre, la révolution russe, la montée du fascisme puis du nazisme, la Deuxième Guerre mondiale, la guerre froide, la décolonisation.

3 Son parcours permet de mieux comprendre les relations complexes entre le féminisme et le pacifisme en France au $\mathrm{XX}^{\mathrm{e}}$ siècle, sujet jusqu'à présent peu traité par l'historiographie française, y compris en histoire des femmes. Et plus encore, de s'interroger sur les ambiguïtés d'un certain pacifisme féminin.

\section{Une stratégie mémorielle, des sources nombreuses}

4 Jeanne Mélin a construit tout au long de sa vie une stratégie mémorielle. Elle a surtout voulu laisser une trace et souligner l'importance de son engagement au sein du mouvement féministe français. Elle a choisi de léguer ses archives personnelles à la bibliothèque féministe de Marie-Louise Bouglé conservée par la Bibliothèque Historique de la Ville de Paris (BHVP) et aux Archives départementales des Ardennes 
(ADA). Par ce geste, elle s'inscrit à la fois dans la mémoire collective nationale du féminisme français mais aussi dans l'histoire de sa région natale.

Dans les 47 boîtes du fonds Mélin de la BHVP, se trouve une correspondance remarquable de 2709 lettres datées de 1905 à 1964, dont celles échangées avec son confident Gustave Fabius de Champville ${ }^{3}$ de 1927 à 1935 : les Lettres à Thalès. Il y a également ses deux romans, des photos, de nombreux documents de toutes sortes (brochures, ${ }^{4}$ coupures de presse, brouillons de discours, d'articles, courriers officiels des associations dont elle a été membre, comptes-rendus des organisations), évoquant son importante activité militante, féministe, pacifiste, communiste, sa profession de foi lors de sa candidature à la présidence de la République en 1947, les comptes-rendus des nombreux congrès pacifistes et féministes auxquels elle a assisté. La BHVP possède aussi des résumés de ses nombreuses conférences. La série 80 des actualités du fonds Bouglé contient un dossier biographique comportant les textes de ses conférences prononcées avant la Grande Guerre ${ }^{5}$. Les archives qu'elle a léguées à son département natal complètent le très riche fonds de la BHVP: outre de précieux documents (brochures, minutes de congrès, photos, etc.), j'ai retrouvé une partie de sa correspondance concernant essentiellement la dernière partie de sa $\mathrm{vie}^{6}$, des manuscrits, un journal intime - composé de 55 cahiers - tenu de 1942 à 1964, une autobiographie manuscrite.

6 Sa bibliothèque personnelle apporte à l'historien des renseignements précieux sur ses lectures, ses influences. Ces documents informent sur les engagements de la fin de sa vie et, plus généralement, sur le Mouvement de l'Abondance de Jacques Duboin, préconisant l'égale répartition des richesses entre tous les citoyens ${ }^{7}$. Cependant, des lacunes subsistent pour la période 1895-1940 : Jeanne Mélin a perdu pendant la Grande Guerre ses archives des années 1890-1914 . Pendant la Deuxième Guerre mondiale, le bâtiment des ADA est incendié à la suite d'un bombardement.

Dans le fonds Gabrielle Duchêne de la Bibliothèque de Documentation Internationale Contemporaine (BDIC), il y a des lettres, des brochures, des comptes-rendus de congrès, de réunions concernant l'activité de Jeanne Mélin à la Ligue Internationale des Femmes pour la Paix et la Liberté (LIFPL). Les archives de la Préfecture de police de Paris ${ }^{9}$ (APP) et les Archives nationales (AN) ${ }^{10}$ possèdent des rapports de police sur le Comité d'Action Suffragiste (CAS), créé par Jeanne Mélin en 1918.

8 J'ai aussi consulté la collection complète de Jus Suffragii ${ }^{11}$ et de la Voix des Femmes ${ }^{12}$ à la bibliothèque Marguerite Durand: on y découvre par exemple le discours de Jeanne Mélin au congrès de Zurich de la LIFPL en 1919. Les documents relatifs au procès d'Hélène Brion $^{13}$, procès où témoigne Jeanne Mélin, se trouvent dans le fonds Hélène Brion conservé par l'Institut Français d'Histoire Sociale et à la bibliothèque Marguerite Durand. Par ailleurs, les archives microfilmées de l'Internationale Communiste, conservées par le Parti Communiste Français, me permettent d'avoir des renseignements à propos de la participation de Jeanne Mélin au Congrès de Marseille de 1921.

9 La consultation de l'inventaire du fonds Cécile Brunschvicg ${ }^{14}$, rassemblant toutes les archives de l'Union Française pour le Suffrage des Femmes (UFSF), déposé au Centre des Archives du Féminisme de la Bibliothèque Universitaire d'Angers, a révélé une similitude d'informations avec le fonds Jeanne Mélin. La même constatation s'impose pour les archives de l'association La Paix par le Droit (APD), conservées aux Archives 
départementales du Gard, ainsi que pour celles de la Ligue des Droits de l'Homme, rendues par la Russie à la France en 2000.

Cet ensemble documentaire révèle ainsi des sources denses et variées: brochures, romans, manuscrits, lettres, journaux intimes, archives des associations dont elle est membre, coupures de presse, sur des sujets variés, dont, par exemple, l'histoire du féminisme français pendant la première moitié du $\mathrm{XX}^{\mathrm{e}}$ siècle.

11 Les lettres sont précieuses pour brosser le portrait de Jeanne Mélin, reconstituer le fonctionnement interne des associations dont elle est membre ou fondatrice, pour recenser ses actions militantes ${ }^{15}$. La plupart d'entre elles sont des lettres adressées à Jeanne Mélin par des figures importantes des courants féministes et pacifistes français (Maria Vérone, Gabrielle Duchêne, Cécile Brunschvicg, Théodore Ruyssen) ou étrangers (Jane Addams, Catherine Marshall). Ces missives traitent en général du féminisme, du pacifisme, de la vie des associations fréquentées ou créées par Jeanne Mélin, des remous provoqués par son adhésion au congrès de La Haye en 1915, de ses solidarités, de ses amitiés et de ses inimitiés. Elles permettent de cartographier ses réseaux, dont les pacifistes de l'APD (Charles Richet, Théodore Ruyssen) et leurs relais régionaux (groupe pacifiste du Poitou) constituent le premier groupe. Parmi les féministes, se distinguent trois autres groupes: les féministes réformistes (Cécile Brunschvicg, Marguerite de Witt-Schlumberger), les féministes radicales parisiennes (Hélène Brion, Gabrielle Duchêne, Henriette Coulmy, Octavie Hasse) et provinciales (Léo Wanner), les féministes étrangères (Aletta Jacobs, Jane Addams, Emily Balch).

Elles permettent aussi de définir la chronologie d'un cheminement qui conduit Jeanne Mélin à affirmer un militantisme avant tout pacifiste, avant de devenir pour une vingtaine d'années (1915-1935) une figure centrale de la LIFPL, puis de tomber dans les dérives d'un pacifisme intégral et l'isolement d'une vieillesse marquée par de « mauvais choix $»^{16}$.

\section{Devenir pacifiste}

Jeanne Mélin est née en 1877 à Carignan (Ardennes) dans une famille bourgeoise. Son père est un industriel anticlérical, républicain, dreyfusard. Il dirige la briqueterie familiale en s'inspirant des théories de Godin. C'est un philanthrope qui souhaite la fin du paupérisme. Jeanne Mélin grandit dans un milieu ouvert, marqué par les horreurs de la guerre de 1870, sensible au jeune mouvement pacifiste. Dans ses Mémoires, elle se souvient: "La guerre toute proche encore était souvent sujet de conversation soit entre soi, soit avec les visiteurs. Tableau effrayant pour la petite fille qui avait tant écouté les conversations des grandes personnes ${ }^{17}$.

Jeanne Mélin reçoit une éducation conforme à celle des jeunes filles de son milieu social au XIX ${ }^{e}$ siècle. Elle fait ses études secondaires au pensionnat Sainte-Chrétienne de Carignan alors que son frère est envoyé au lycée de Charleville. Bien qu'anticléricale, sa famille respecte les normes sociales en vigueur. La classe sociale prédomine sur le sexe. Jeanne Mélin regrette, des années plus tard, dans ses Mémoires et dans ses Lettres à Thalès, de ne pas avoir reçu d'éducation scientifique et de ne pas avoir appris de langues étrangères. Pendant l'adolescence, elle perd la foi et se découvre une conscience anticléricale, libre-penseuse, renforcée par la lecture de Victor Hugo et de George Sand. 
15 En 1898, elle découvre dans le Petit Ardennais un appel de Sylvie Hugo-Flammarion faisant la promotion de l'association La Paix et le Désarmement par les Femmes. Jeanne Mélin crée un groupe local de cette organisation dans les Ardennes et y développe un discours modéré, basé sur les vertus pacificatrices et éducatrices de la maternité. Sa réflexion pacifiste naît du refus de voir revenir la guerre. Elle oppose le masculin, symbole de violence au féminin, symbole de vie. Elle est très influencée par la pensée positiviste du XIX ${ }^{\mathrm{e}}$ siècle, persuadée que la science symbole de progrès et de paix ne peut être utilisée à des fins guerrières. De 1900 à 1914, elle milite en faveur d'un pacifisme modéré, adhérant d'abord à l'association la Paix par le Droit (1901), puis fondant une antenne ardennaise de cette société pacifiste (1908). Dans ce cadre, elle milite en faveur de l'arbitrage et d'un code international des nations. Elle participe aux congrès pacifistes nationaux (congrès de l'APD de la Rochelle de 1908, le $6^{e}$ congrès national des Sociétés Françaises de la Paix de Reims en 1909), et internationaux (le $18^{\mathrm{e}}$ congrès universel de la Paix de Londres de 1908, le $19^{e}$ congrès universel de la Paix de Stockholm de 1910). Elle se construit un réseau pacifiste national - l'APD - et international - Bertha Von Suttner ${ }^{18}$, Alfred Fried ${ }^{19}$.

16 Elle souhaite l'émergence d'une éducation de la paix dans les écoles. En 1907, répondant à un concours du journal Le Matin sur l'éducation idéale du citoyen, elle revisite l'enseignement de l'histoire, préconisant l'enseignement de l'histoire des sciences plutôt que celle des batailles. Elle cherche à éradiquer la violence, non seulement au niveau de l'État, mais aussi à l'échelle des individus, cherchant à faire disparaitre la violence chez les enfants. Elle élabore un système de coéducation ${ }^{20}$ basé sur l'échange des qualités masculines et féminines. Par exemple, « les garçons seraient obligés de s'adoucir pour vivre avec leurs [futures] compagnes [...] $»^{21}$. Quant aux filles, «elles deviendraient plus viriles, moins fragiles et moins peureuses" et sauraient mieux se défendre face aux difficultés de la vie ${ }^{22}$.

Grâce au pacifisme, Jeanne Mélin découvre les idées féministes, résultat d'« une éducation féminine par la paix $»^{23}$, selon l'expression de l'historien Daniel Armogathe : «En s'ouvrant à une analyse internationale des luttes, elle découvre une solidarité de sexe qu'elle n'aurait pas rencontrée en d'autres temps $»^{24}$.

L'un des objectifs de ce travail est d'étudier de quelle façon s'articule l'appropriation par Jeanne Mélin des concepts d'identité féminine, de masculinité et de différence des sexes. Ses constantes références à la maternité pacificatrice ne risquent-t-elles pas en effet d'obscurcir ses convictions féministes, de les rendre moins lisibles, de les dénaturer?

19 Jeanne Mélin est avant tout une pacifiste féministe, pour qui le féminisme est un vecteur de paix. Elle base sa réflexion sur la transmission des valeurs de paix, par les femmes, porteuses de vie. Le droit de vote des femmes est avant tout le sésame de la paix : les femmes donnent la vie et, par conséquent, sont dans l'incapacité naturelle de soutenir une politique guerrière. Elle n'en déroge pas tout au long de sa vie. Selon elle, obtenir le droit de vote au nom de la paix n'est pas une stratégie de reconnaissance politique, comme le souhaitent certaines féministes. C'est au contraire la seule opportunité pour mener à bien son engagement pacifiste. En 1906, elle adhère à la SFIO, influencée par les pensées pacifistes et socialistes de Jean Jaurès. Elle milite aussi à la Libre-Pensée et à la Ligue des Droits de l'Homme. Cette multi-appartenance à plusieurs sociétés est une caractéristique des féministes de sa génération. Elle s'inscrit également aussi dans le courant féministe de son époque en fondant, en 1912, le groupe de l'Union 
Française pour le Suffrage des Femmes (UFSF) des Ardennes. Elle y revendique le droit de vote par étapes et le suffrage municipal. Dans les réunions qu'elle organise, elle insiste sur les qualités dites féminines bénéfiques pour la politique. Par exemple, la présence des femmes dans les conseils municipaux permettrait d'atténuer les fléaux sociaux. Jeanne Mélin milite en faveur d'une égalité des sexes respectueuse des différences sexuées.

Ainsi, elle acquiert une solide réputation de conférencière pacifiste et féministe reconnue en France et en Europe entre 1910 et 1914. En novembre-décembre 1913, elle fait une tournée de 30 conférences en France (Grand Ouest, région lyonnaise, Jura) pour la paix et le suffrage des femmes. Ses qualités oratoires sont soulignées par les journalistes qui suivent ses conférences, comme, par exemple, dans le Poitou en décembre 1913. Un journaliste de La Fraternité, journal républicain du Poitou, écrit le 27 décembre 1913 : «Sa grâce, son humour, sa voix bien timbrée, sa parfaite connaissance des questions sociales et politiques, [...] tout en cette prophétesse de la Paix concourut à l'éclatant succès des 12 manifestations dont elle fut l'âme pathétique $»^{25}$. En janvier 1914 elle voyage en Belgique et aux Pays-Bas pour y tenir des réunions sur le même thème.

21 En 1913, c'est une pacifiste pensant concilier l'arbitrage, jugé inefficace et bourgeois par la SFIO, et les réflexions socialistes sur la paix. Dans son argumentaire pacifiste, elle introduit peu à peu la notion de responsabilité guerrière du capitalisme. Elle soutient Jaurès mais ne partage pas les théories antimilitaristes de Gustave Hervé. Pendant la Grande Guerre, elle rejoint les maigres troupes des féministes radicales et revendique le droit de vote intégral des femmes, durcissant son discours féministe pour tenter de mettre fin à ce conflit meurtrier.

\section{Le pacifisme radical d'une militante reconnue et respectée}

En 1914, les premières divergences avec l'UFSF apparaissent. Pendant la Grande Guerre, Jeanne Mélin s'attire les foudres des autorités françaises, en exigeant le droit de vote pour les Françaises afin de mettre un terme aux combats. Refusant l'Union Sacrée, elle regrette l'absence de l'UFSF au congrès de La Haye ${ }^{26}$ auquel elle donne son adhésion. Elle se rapproche des féministes hollandaises (Aletta Jacobs), des futures fondatrices de la Ligue Internationale des Femmes pour la Paix et la Liberté (Jane Addams), des féministes radicales (Hélène Brion, Gabrielle Duchêne). Elle utilise sa correspondance comme une arme militante en se faisant le relais français du congrès de La Haye. En 1918, Jeanne Mélin fonde le Comité d'Action Suffragiste prônant le vote intégral. Elle témoigne au procès d'Hélène Brion, accusée de faire de la propagande pacifiste. Les deux femmes sont convaincues de l'importance de la participation active des femmes à la construction du monde futur, portant toutes deux de grands espoirs en la politique étrangère du président américain Wilson. Toutefois, Hélène Brion, à la différence de Jeanne Mélin qui est une pacifiste féministe, se définit comme une féministe pacifiste. Se démarquant de Jeanne Mélin, qui ne parvient pas à dépasser le lien intellectuel entre l'éternel féminin, la paix et le suffrage des femmes, elle est persuadée que l'éternel féminin est un mythe, que les femmes, comme les hommes, ont des pulsions violentes, des instincts défensifs. Par la codification de la guerre, les hommes, dit-elle, se sont 
appropriés la violence et ont dépossédé les femmes de cette obligation de se battre. Devant ses juges, elle regrette que les femmes ne puissent pas se battre.

Du pacifisme modéré, respectueux de la patrie et reposant sur l'arbitrage, dont elle se fait la propagandiste de 1900 à 1914, Jeanne Mélin évolue dans les années 1920 vers un pacifisme radical, qui entend empêcher le retour de la guerre et fonder un internationalisme plus efficace que celui de la SDN, puis vers un pacifisme intégral dont elle se réclame pour la première fois en 1931. De 1919 à 1925, elle axe son action militante sur les relations internationales, comme en témoignent les correspondances conservées. Entre 1915 et 1924, elle reçoit 852 lettres émanant notamment de féministes radicales (Gabrielle Duchêne, Louise Bodin, l'une des journalistes de La Voix des Femmes), de dirigeantes de la LIFPL (Jane Addams, présidente, Chrystal Mac Millan, Gertrud Baer et Lida Gustava Heymann, membres de la section allemande).

Comment Jeanne Mélin intègre-t-elle ses convictions communistes à sa réflexion féministe et pacifiste? De 1920 à 1923, elle est membre du PCF persuadée que le féminisme et le communisme sont compatibles. L'idéal communiste l'attire, elle y voit un espoir de paix mondiale et de suffrage féminin généralisé. Mais en janvier 1923 elle démissionne du Parti : la fédération des Ardennes où elle milite n'admet pas son appartenance à la LIFPL. Sa déception est profonde.

De 1919 à 1925, elle milite très activement pour la paix et pour le droit de vote des femmes, particulièrement au sein de la LIFPL. Elle refuse le traité de Versailles, et notamment la thèse de la culpabilité allemande. Elle multiplie les gestes médiatiques et symboliques en faveur du rapprochement franco-allemand. Au congrès fondateur de la LIFPL, à Zurich, en 1919, elle marque les esprits en serrant la main de la déléguée allemande Lida Gustava Heymann. Dans son discours, elle n'admet pas que la France humilie l'Allemagne par les traités de paix. Elle se proclame sœur de ces femmes qui, comme elle, ont lutté contre tous les impérialismes et tous les militarismes.

Jeanne Mélin voyage beaucoup en Europe pour la LIFPL. Elle est à Bruxelles et à Liège en 1920 pour lancer la section belge, à Vienne en 1921. Elle participe activement à la construction des réseaux européens et internationaux des féministes pacifistes. L'influence réelle de ces réseaux est difficile à évaluer. Par exemple, en décembre 1922, la délégation des Messagères de la Paix, composée de Jane Addams, de Catherine Marshall et de Jeanne Mélin, reçoit le soutien des pays scandinaves contre l'occupation française de la Ruhr. Malheureusement, leur neutralité leur interdit de faire pression sur la France pour empêcher l'occupation de la Ruhr et demander la re-discussion des traités. Les déléguées proposent la création d'un emprunt international pour mettre fin aux réparations. Elles n'obtiennent pas le même succès à Londres et à Paris où les gouvernements français et britannique ne les reçoivent pas.

La modernité de leurs propositions n'est pas entendue. Jeanne Mélin, dans Les Cahiers de la Paix, rédigés pour le congrès de Washington de la LIFPL, en 1924, souhaite la modification profonde de la constitution de la SDN, afin que soient établis en priorité «les droits et les devoirs internationaux des peuples». Sous l'influence de l'internationalisme, de la coopération, de la supranationalité, Jeanne Mélin souhaite l'émergence d'un "nouveau régime international » entraînant "une réorganisation totale du monde ». Ces réseaux féministes prennent part, à leur manière, à la vie diplomatique internationale bien qu'en étant exclus officiellement. Cette occultation officielle explique leur non-visibilité dans l'histoire. Par l'exemple de la conférence d'été de Honfleur, co-organisée - à son initiative personnelle - avec la philanthrope 
américaine Mary Kelsey, Jeanne Mélin participe à la construction d'un mouvement internationaliste féministe et pacifiste. Pendant les étés 1923, 1924, 1925, des jeunes femmes et hommes du monde entier se réunissent à Honfleur. Ces rencontres doivent favoriser la coopération intellectuelle entre les nations, soustraire les jeunes générations à l'emprise de la guerre en dissipant les malentendus véhiculés par les propagandes nationales. Les initiatrices souhaitent que la SDN soit remplacée par une société des peuples internationaliste, prônant l'égalité des sexes et l'indépendance économique des femmes, le partage équitable des richesses, le respect des minorités.

À partir de 1926, Jeanne Mélin connaît une longue période de retrait, entrecoupée de résurgences militantes. Mais elle continue de correspondre et de tisser son réseau. Entre 1925 et 1934, elle a reçu au moins 1352 lettres de France et d'ailleurs : lettres de Cécile Brunschvig, de Séverine, de Madeleine Vernet, la fondatrice de la «Mère éducatrice » notamment. Entre 1927 et 1930, l'écriture devient un refuge pour fuir une situation familiale difficile.

Elle diversifie les modes d'expression écrite : les romans pour l'opinion publique, les Lettres à Thalès pour son introspection personnelle. Dans la tradition des romanciers prolétariens, elle souhaite toucher le peuple autrement et lui transmettre ses convictions. Le roman Jean ou à travers la misère (1927) ${ }^{27}$ est un hommage vibrant à Jean Jaurès. Dans son roman Marceline en vacances (1929) ${ }^{28}$, elle milite en faveur du contrôle des naissances dans un souci pacifiste: l'éducation sexuelle des jeunes filles et des jeunes hommes, l'avortement, la maîtrise des naissances. Elle veut responsabiliser les jeunes, surtout les garçons, qui ne sont pas exclus de son projet. Ces réflexions ne sont pas nouvelles pour Jeanne Mélin. Déjà, en 1907, dans son projet éducatif pour le concours du journal Le Matin, elle militait dans un souci néo-malthusien pour le contrôle des naissances et l'éducation sexuelle.

Dans les Lettres à Thalès, elle fait part de son état d'esprit, de ses réactions à l'actualité, de ses réflexions féministes et pacifistes. Plusieurs thèmes traversent l'ensemble de ses textes: la paix, le droit de vote des femmes, la revendication du contrôle des naissances. Les lettres à Thalès étaient le brouillon de ses romans. Leur lecture est ardue. Dans une même page, Jeanne Mélin est capable de mêler toutes ses idées, sans enchaînement logique, ce qui rend leur compréhension difficile. Ses tourments, voire ses angoisses, sont manifestes. En effet, elle commence ces missives pendant l'agonie de sa mère au début de 1927 et témoigne de sa profonde solitude affective.

En mars 1927, elle démissionne du conseil consultatif de la LIFPL et, en décembre 1927, elle envisage même de démissionner définitivement de la LIFPL et commence à rédiger une lettre à Gabrielle Duchêne. Un conflit idéologique avec Gabrielle Duchêne est à l'origine de cette initiative. En effet, cette dernière devient une compagne de route du PCF, après un voyage en URSS en 1927, et intensifie alors la politique anti-impérialiste de la section française de la LIFPL. Gabrielle Duchêne ne semble pas avoir apprécié le choix de Jeanne Mélin d'avoir invité, pour une conférence, l'écrivain allemand Heinrich Mann, auteur du roman, Le Professeur Umrat. ${ }^{29}$ Finalement Jeanne Mélin n'envoie pas sa lettre à Gabrielle Duchêne et ne quitte pas la LIFPL ${ }^{30}$. En créant, en 1930, le Cercle Pax Occident-Orient (CPOO), elle cherche à unifier les sociétés pacifistes françaises pour qu'elles aient plus de poids face à la menace de guerre. Le 11 novembre 1931, le CPOO organise une parade de la paix. Ce défilé, rassemblant 19 sociétés oeuvrant pour la paix, traverse Paris en autobus et marque deux arrêts symboliques à l'Étoile, sur la tombe du soldat inconnu, puis, au pied de la 
Tour Eiffel ${ }^{31}$. Jeanne Mélin tombe dans une utopie pacifiste. En 1933, par exemple, elle adresse un manifeste au peuple allemand pour lui proposer une fédération économique européenne. En 1933, elle ne dénonce pas le nazisme. Après 1936, elle se retire de la vie publique. Elle connaît des soucis de santé et sa vie privée est difficile. Elle se remet difficilement de son bref mariage, de son divorce en 1934 et du suicide de son frère la même année. Elle a totalement sacrifié sa vie de femme à son militantisme mais aussi à sa famille, à laquelle elle était totalement dévouée. Sa solitude psychologique est grande et elle ne parvient pas à la contrôler.

\section{Dérives et isolement}

À la fin des années 1930, son pacifisme intégral lui met des œillères et l'entraîne vers un comportement à double tranchant : elle discerne tardivement le danger du fascisme et du nazisme. En 1935, elle participe à quelques réunions du Comité Mondial des Femmes contre la Guerre et le Fascisme, créé en 1934 par Gabrielle Duchêne. Jeanne Mélin voit dans ce comité un moyen de revitaliser le mouvement féministe des années 1930 très divisé. Elle est persuadée que, seule l'union des féministes rassemblées dans un sentiment internationaliste, va permettre de combattre la guerre et le fascisme. Elle s'exprime peu sur la guerre d'Espagne, n'écrivant qu'un poème en décembre $1936 . \mathrm{Ne}$ prenant parti pour aucun des deux camps, elle établit la prééminence du «droit à la vie, base du droit de l'homme ${ }^{32}$. Ces phrases sont sans équivoque $:$ refusant que le sang coule, elle semble ne pas accepter l'idée même de la guerre d'Espagne.

Dans ses Mémoires, Jeanne Mélin, en 1957, témoigne de son désespoir et de son isolement lorsqu'elle aborde son souvenir de la déclaration de guerre : "Alors cette fois, décrire ma plus grande désillusion m'est impossible ${ }^{33}$... quoi, me disais-je, avoir tant travaillé, sans relâche, en faveur de l'entente entre les peuples, de la Paix et de la fraternité humaine et assister, impuissante à l'effondrement d'un si haut idéal ! $\aleph^{34}$ Dans ce même texte, elle tente de cacher un lourd secret : la dérive de son pacifisme intégral parce que « Résistante à la guerre $»^{35}$ vers le soutien au régime de Vichy.

En novembre 1940, reprenant le discours de l'extrême-droite dans un poème pamphlétaire, intitulé La Salaucratie ${ }^{36}$, elle s'attaque violemment aux radicaux dont elle était proche : elle les accuse de cupidité, de faire main basse sur l'exécutif et le législatif sans se soucier des électeurs. Autre cliché de la droite conservatrice, elle leur reproche d'avoir entraîné la France dans la Deuxième Guerre mondiale.

Elle adhère idéologiquement au triptyque "Travail, Famille, Patrie ». Cette foi en la terre, en la régénérescence de la nation n'est pas une idée nouvelle pour Jeanne Mélin qui a des antécédents conservateurs. En 1907, dans un programme éducatif, elle faisait déjà l'apologie de la maternité à la campagne, symbole de la renaissance de la France. Elle reprend ainsi une partie du discours vichyste valorisant l'éternel féminin, la maternité, garante de la tradition, de l'éternité, de la rédemption, rempart contre l'individualisme et le désordre.

37 C'est dans cette optique qu'elle soutient ouvertement la rencontre de Montoire du 24 octobre 1940. Dans un poème, Montoire, daté du 3 février 1941, elle se dit persuadée que cette rencontre peut mettre fin à la politique des armements et par conséquent à la guerre $^{37}$. S'inspirant du discours collaborationniste de Marcel Déat et de Jacques Doriot, elle accuse la City londonienne d'attiser le climat guerrier, d'être la seule responsable 
de la guerre. Elle s'attaque aux Juifs en reprenant les dires antisémites les plus scabreux sur l'argent, mentionnant, par exemple, en décembre 1940 «la bataille pour l'or ${ }^{38}$.

Le pacifisme intégral ne doit faire aucune concession à la guerre. Donc, Jeanne Mélin refuse tout acte de résistance parce qu'il peut, selon elle, réactiver la guerre. En septembre 1942, dans le poème Guerre Civile, elle écrit : «Leur mot d'ordre : la guerre civile. Répandre et de façon habile. Jette la patrie dans un bourbier $»^{39}$.

L'évolution de Jeanne Mélin sous l'Occupation est à première vue contradictoire: apôtre de la révision du traité de Versailles et de la réconciliation franco-allemande dans les années 1920, sympathisante du Comité Mondial des Femmes contre la Guerre et le Fascisme dans les années 1930, elle prend la direction opposée. Elle s'enlise dans le soutien intellectuel à la collaboration en tenant son journal intime. Ses idées féministes ne sont pas à l'origine de ce dévoiement. Elle est très influencée par la pensée pacifiste de la fin du XIX ${ }^{\mathrm{e}}$ siècle : elle reste persuadée toute sa vie que la science est symbole de progrès et de paix. Ses désillusions sont cruelles. Elle rejoint ce que Philippe Burin appelle la "gauche collaboratrice $»^{40}$ composée de pacifistes intégraux. Ses engagements collaborationnistes ont peu de portée publique. C'est pourquoi elle n'est pas touchée par l'épuration.

Ce parcours entre ombres et lumières, entre innovation et conservatisme se termine sur une fin de vie solitaire. Après la guerre, Jeanne Mélin se renferme dans une utopie profonde dont l'axe nodal semble être l'abolition du salariat et le partage des richesses. Elle rejoint les rangs du Mouvement Français pour l'Abondance, créé par Jacques Duboin, un ancien ministre des finances d'Aristide Briand, qui développe ces théories. Auparavant, en 1947, elle se présente à la présidence de la République et revendique la parité pour assurer une politique pacifiste. Avant elle, Hubertine Auclert avait aussi milité en faveur de cette cause.

Comme pour la revendication du contrôle des naissances en 1928, elle agit en franctireur, au moment où les réseaux féministes déclinent. Sa candidature attire peu la presse malgré un contexte favorable aux femmes. Trois ans auparavant, elles avaient obtenu le droit de vote. Son indépendance, son refus des partis la desservent une nouvelle fois.

Après 1947, sa réflexion féministe se tarit. Dans les années 1950, elle préfère suivre avec passion l'actualité des relations internationales. Dans un souci pacifiste, en 1959, elle se prononce en faveur du birth control. Sur ce point, elle partage les préoccupations de la nouvelle génération des féministes qui, en 1956, viennent de créer Maternité Heureuse. Ses préoccupations ne sont plus seulement pacifistes. Ses revendications de 1928 sont récurrentes : elle milite pour la reconnaissance de la sexualité et du plaisir féminin. Comme Simone de Beauvoir, elle condamne la soumission de la femme dans le rapport sexuel. Malheureusement, Jeanne Mélin tient ces propos alors qu'elle est totalement marginalisée et coupée du noyau féministe qui renaît dans les années 1950. En 1928, elle était aussi isolée, appartenant à la minorité des féministes œuvrant pour le contrôle des naissances. Par souci de reconnaissance du monde littéraire, elle publie ses romans sous un nom d'emprunt, ce qui fragilise la portée de son discours.

Pendant la guerre froide, parvient-elle à intérioriser le discours pacifiste spécifique aux années 1950 et 1960 (dont les axes majeurs sont la conférence de Wroclaw, l'appel de Stockholm) face au risque de guerre nucléaire? Sa fibre pacifiste est primordiale dans les années à cette époque. Membre du Mouvement de la Paix, et de l'appel de Stockholm, par principe, sans militer effectivement, elle s'oppose au danger de la 
guerre atomique en noircissant des pages entières de son journal intime. Elle est très lucide sur l'inutilité de cette démarche, n'ayant plus de tribune militante ${ }^{41}$. Elle refuse la guerre froide. Elle y voit l'échec de son engagement pacifiste qui tend alors vers le socialisme utopique, développant une réflexion sur l'abolition du salariat, le partage des richesses.

L'examen de son cheminement ultime face aux mouvements et guerres de décolonisation, notamment celle d'Algérie, montre comment Jeanne Mélin parvient à établir un lien entre son engagement pacifiste et la décolonisation pour laquelle elle se prononce. Très influencée par les théories non-violentes du mahatma Gandhi, elle aurait aimé que l'indépendance de l'Algérie ne résulte pas d'une guerre.

Dans les derniers mois de sa vie, l'anticléricalisme acharné de sa lointaine jeunesse laisse curieusement la place à un état d'esprit conforté par les valeurs progressistes portées par Vatican II qui l'inspirent désormais. Elle salue l'encyclique Pacem in terris, proclamée le 11 avril 1963, ordonnant la paix entre toutes les nations, fondée sur la vérité, la justice, la charité, la liberté. Elle y voit l'aboutissement de sa vie de militante pacifiste. Jeanne Mélin aurait apprécié, dans le prolongement de Vatican II qu'elle a soutenu, malgré des interrogations provoquées par son anticléricalisme viscéral, la déclaration du pape Paul VI du 23 juin 1964 où il annonce la création d'une commission pontificale sur le contrôle des naissances.

Jeanne Mélin meurt en 1964 dans sa ville natale de Carignan. Malgré l'importance de son engagement, elle n'est pas passée à la postérité. Son isolement, puis, par conséquent, son absence de la scène mémorielle française ne reflète-t-il pas une caractéristique de la première vague du féminisme, qui, comme le rappelle Christine Bard, est dans les années 1930, "sclérosé", "affaibli par des années de luttes insatisfaites $»^{42}$ ? Ne souhaitant pas ternir l'image d'apôtre de la paix et du féminisme qu'elle a savamment construite pendant des années, se sachant pendant l'Occupation à contre-courant des autres féministes, qui, dans leur grande majorité, ont laissé de côté leurs engagements pacifistes pour combattre le régime de Vichy ${ }^{43}$, Jeanne Melin a volontairement cherché à occulter cette zone d'ombre de sa vie, c'est peut-être pour cette raison que l'histoire l'a oubliée.

\section{BIBLIOGRAPHIE}

ANTOINE Régis, 2002, La Littérature pacifiste et internationaliste française : 1919-1935, Paris, L'Harmattan.

ARMOGATHE Daniel, 1984, « Les Femmes et la paix en France aux XIX ${ }^{\mathrm{e}}$ et XX $\mathrm{XX}^{\mathrm{e}}$ siècles : figure d'une problématique ", in Le Bricquir Danielle, Thomas Odette (dir.), Féminisme et pacifisme : même combat, Paris, Les Belles Lettres, pp. 43-51.

AUDOIN-ROUZEAU Stéphane, Annette Becker, 2000, Retrouver la guerre : 14-18, Paris, Gallimard. AUDOIN-ROUZEAU Stéphane, Annette Becker, Christian Ingrao, Henry Rousso (dir.), 2003, La Violence de guerre : 1914-1945, Bruxelles, Complexe. 
BARD Christine, 1995, Les Filles de Marianne, Paris, Fayard.

--, 1997, « Le dilemme des féministes françaises face au nazisme et à la menace de guerre (1933-1940) », in Liliane Kandel, Féminismes et nazisme, Publications de l'Université Paris 7 Denis Diderot, pp. 148-160.

BAUDORRE Philippe, 1995, Barbusse. Le pourfendeur de la Grande Guerre, Paris, Flammarion. BIONDI Jean-Pierre, 2000, La Mêlée des pacifistes : la grande dérive, Paris, Maisonneuve et Larose. BLUM Françoise, 1982, Mouvements de Femmes contre la guerre (1915-1935) : essai d'analyse d'un féminisme pacifiste, Paris, Université Paris 1.

--, 1985, « Le Féminisme sous la III ${ }^{e}$ République : 1914-1939. L'impact de la guerre sur les mouvements de femmes », Matériaux pour l'histoire de notre temps, $\mathrm{n}^{\circ} 1, \mathrm{pp} .12-15$.

BURRIN Philippe, 1986, La Dérive fasciste, Doriot, Déat, Bergery, Paris, Seuil.

CAPDEVILA Luc, ROUQUET François, VIRGILI Fabrice, VOLDMAN Danièle, 2003, Hommes et femmes dans la France en guerre : 1914-1945, Paris, Payot-Rivages.

CHAPERON Sylvie, 2000, Les Années Beauvoir : 1945-1970, Paris, Fayard.

CHICKERING Roger, 1975, Imperial Germany and a World without war, Princeton, Princeton University Press.

COVA Anne, 1997, Maternité et droits des femmes en France : XIX ${ }^{\mathrm{e}}-\mathrm{XX}^{\mathrm{e}}$ siècles, Paris, Anthropos.

DREYFUS Michel, 1981, « La Ligue Internationale des Femmes pour la Paix et la Liberté », Les Cahiers du Féminisme, ${ }^{\circ} 18$, pp.47-51.

--, 1992, « Des femmes pacifistes durant la décennie des années 1930 », Matériaux pour l'histoire de notre temps, $\mathrm{n}^{\circ} 30, \mathrm{pp} .32-53$.

FRAISSE Geneviève, 1976, «Et si les mères désertaient la guerre... Madeleine Vernet (1879-1854) », Les Cahiers du GRIF, n 14 et 15.

GROSSI Verdiana, 1994, Le Pacifisme européen, Bruxelles, Bruylant.

HIRATA Helena, LABORIE Françoise, LE DOATE Hélène, SENOTIER Danièle (dir.), 2000, Dictionnaire critique du féminisme, Paris, PUF.

INGRAM Norman, 1991, The Politics of dissent pacifism in France, Oxford, Clarendon Press.

KLEJMAN Laurence, ROCHEFORT Florence, 1989, L'Égalité en marche. Le féminisme sous la III ${ }^{\mathrm{e}}$ République, Paris, Editions des Femmes/ Presses de la Fondation Nationale des Sciences Politiques.

KNIEBIEHLER Yvonne, 1982, Histoire des Mères du Moyen-Âge à nos jours, Paris, Hachette. LORRAIN Sophie, 1999, Des Pacifistes français et allemands, pionniers de l'entente francoallemande : 1870-1925, Paris, L'Harmattan.

MUEL-DREYFUS Francine, 1996, Vichy et l'éternel féminin, Paris, Seuil. OFFEN Karen, 2000, European Feminisms, 1700-1750, Stanford, Stanford University Press. OFFENSTADT Nicolas, OLIVERA Philippe, 1993a, «L'Engagement pour la Paix : un ou des pacifismes », Matériaux pour l'histoire de notre temps, $n^{\circ}$ 30, pp. 54-56.

--, 1993b, « L'Engagement pacifiste en France 1919-1939 », Bulletin d'histoire du temps présent, $\mathrm{n}^{\circ}$ 51, pp. 47-93. 
RIOT-SARCEY Michèle, 2002, Histoire du féminisme, Paris, La Découverte.

SCOTT Joan Wallace, 1988a, Gender and the Politics of History, New York, Columbia University Press.

--, 1988b, « Genre. Catégorie d'analyse », Les Cahiers du GRIF, n 37-38.

VAÏSSE Maurice, 1993, Le Pacifisme en Europe des années 1920 aux années 1950, Bruxelles, Bruylant.

VILLEPIN (de) Patrice, 1988, « Plutôt la servitude que la guerre! Le pacifisme intégral dans les années trente », Relations Internationales, $n^{\circ} 53$, pp.53-67.

TARDIVEL Jacqueline, 1993, « Des Pacifistes aux Résistantes, les femmes et le communisme entre les deux guerres ", thèse d'histoire sous la direction de Michelle Perrot, Université Paris 7 Denis Diderot.

THEBAUD Françoise (dir.), 1991, Histoire des Femmes, le XX ${ }^{\mathrm{e}}$ siècle, Paris, Plon.

--, 1998, Écrire l'Histoire des Femmes, Paris, ENS.

THEBAUD Françoise, ZANCARINI-FOURNEL Michelle, « Éditorial », CLIO, Histoire, Femmes et Sociétés, $\mathrm{n}^{\circ} 18$.

VAHE Isabelle, 2004, « Jeanne Mélin (1877-1964), un parcours singulier dans la mouvance féministe et pacifiste en France au XXe siècle ", thèse d'histoire sous la direction de Yannick Ripa, Université Paris 8.

\section{NOTES}

1. Vahé 2004.

2. Thébaud $1991: 13$.

3. Née en 1865 à Paris, c'est un littérateur prolixe, membre du comité exécutif du parti radical. Il dirige divers journaux dont Le Grand National, Le Fermier, Le Bulletin Quotidien de la Bourse du Commerce. Il est l'auteur d'ouvrages de vulgarisation agricole, de livres sur la magie et les sciences occultes.

4. Par exemple, une brochure de 1925 de Jeanne Mélin consacrée au droit de vote des femmes. BHVP, fonds Bouglé, fonds Jeanne Mélin, boîte 24. Jeanne Mélin, Le Suffrage des femmes: ses conséquences sociales et économiques, éditions Henri Durville, 1925, 6 p.

5. BHVP, fonds Bouglé, série actualités 80 , dossier biographique Jeanne Mélin.

6. De 1940 à 1964.

7. ADA, collection Jeanne Mélin, $15 \mathrm{~J} 13$.

8. C'est par exemple le cas de sa correspondance avec le couple Flammarion.

9. APP,Ba 1651.

10. AN, F7.

11. Jus Suffragii est le journal de l'Association Internationale pour le Suffrage des Femmes.

12. La Voix des Femmes, dont le premier numéro paraît en 1917, accueille dans ses

colonnes les articles de féministes radicales pacifistes (Colette Reynaud, Séverine, Hélène Brion).

13. Jeanne Mélin témoigne à ce procès.

14. Ce fonds, saisi par les Allemands en 1940, puis récupéré par les Soviétiques en 1945, est revenu en France en 2000.

15. Comme par exemple l'action de lobby en faveur du suffrage féminin auprès des parlementaires. 
16. Jeanne Mélin a reçu entre 1905 et 19642673 lettres qui se répartissent de la façon suivante (1905-1914 : 88 lettres, 1915-1924 : 852 lettres, 1925-1934 : 1352 lettres, 1935-1944 : 224 lettres, 1945-1964 : 38 lettres). Après 1914, les lettres révèlent au lecteur l'élargissement du cercle des relations de Jeanne Mélin qui compte tous les noms importants des milieux féministes et pacifistes français. Sa correspondance permet aussi de détecter les failles de son parcours, les ruptures de ton de son récit de vie.

17. ADA, collection Jeanne Mélin, 15 J 1, Jeanne Mélin, Mémoires, pp. 1-2.

18. Bertha Von Suttner (1843-1914), prix Nobel de la Paix en 1905, est l'auteure de Bas les Armes (1889) qui popularise le pacifisme auprès du grand public.

19. Alfred Fried (1864-1921) est une personnalité très importante du mouvement pacifiste allemand. Prix Nobel de la Paix en 1909, il théorise le pacifisme révolutionnaire dont la priorité est de découvrir les facteurs déclenchant les guerres avant même de penser à leur prévention.

20. La coéducation est, selon le Littré de 1877, «l'éducation en commun ». En 1905, Paul Robin, lors du congrès des Amicales des Instituteurs et Institutrices, définit la "coéducation » comme un « choix de société pour construire des rapports harmonieux et égalitaires selon les hommes et les femmes ». Voir Thébaud, Zancarini-Fournel $2003: 18$.

21. BHVP, fonds Bouglé, fonds Jeanne Mélin, cahier manuscrit de Jeanne Mélin, contribution au concours national du journal Le Matin, février 1907.

22. BHVP, fonds Bouglé, fonds Jeanne Mélin, cahier manuscrit de Jeanne Mélin, contribution au concours national du journal Le Matin, février 1907.

23. Armogathe $1984: 43-51$.

24. Armogathe $1984: 43-51$.

25. BHVP, fonds Bouglé, actualités 80, dossier biographique Jeanne Mélin, D 527, La Fraternité de Lezay, 27 décembre 1913.

26. Le congrès international des Femmes pour la Paix de La Haye est organisé par des féministes hollandaises et anglaises. Il se propose de régler les conflits mondiaux par des moyens pacifiques et par l'extension mondiale du suffrage féminin.

27. Jeanne Mélin, Jean ou à travers la misère, 1927.

28. Jeanne Mélin, Marceline en vacances, 1929.

29. BHVP, fonds Bouglé, fonds Jeanne Mélin, boîte 44, lettre de Jeanne Mélin à Gabrielle Duchêne, 12 décembre 1928.

30. BHVP, fonds Bouglé, fonds Jeanne Mélin, boîte 2, Jeanne Mélin, Lettres à Thalès, 15 décembre 1927, 14 janvier 1927.

31. BHVP, Fonds Bouglé, fonds Jeanne Mélin, boîte 31, L'Echo du IXe arrondissement, 18 novembre 1931.

32. BHVP, fonds Bouglé, fonds Jeanne Mélin, boîte 47(1), texte manuscrit de Jeanne Mélin, Aux Petits des humains, 13 décembre 1936.

33. Ce mot est souligné par Jeanne Mélin .

34. ADA, collection Jeanne Mélin, $15 \mathrm{~J}$ 1, Jeanne Mélin, Mémoires, p. 197.

35. ADA, collection Jeanne Mélin, 15 J 1, Jeanne Mélin, Mémoires, pp. 200- 201.

36. BHVP, fonds Bouglé, fonds Jeanne Mélin, boîte 22, Jeanne Mélin, La Salaucratie, 22 février 1941.

37. BHVP, fonds Bouglé, fonds Jeanne Mélin, boîte 22, Jeanne Mélin, Montoire, 3 janvier 1941.

38. BHVP, fonds Bouglé, fonds Jeanne Mélin, boîte 22, Jeanne Mélin, La monnaie, 12 et 13 décembre 1940.

39. ADA, collection Jeanne Mélin, 15 J 2, Jeanne Mélin, Au jour le jour, journal intime, Guerre Civile, 20 septembre 1942.

40. Burrin $1986: 448$.

41. ADA, collection Jeanne Mélin, 15 J 3 (1), Libres Propos ardennais, 28 septembre 1958.

42. Bard 1997 : 148. 


\section{RÉSUMÉS}

L'intérêt de ma thèse est d'étudier les relations entre le féminisme et le pacifisme en France au $X^{\text {e }}$ siècle, sous l'angle de la biographie de Jeanne Mélin (1877-1964), pacifiste, féministe, écrivaine. Le sens de cette recherche est de souligner l'ambiguïté de la mise en perspective par Jeanne Mélin du féminisme et du pacifisme, d'étudier son appropriation des concepts d'identité féminine, de masculinité et de différence des sexes. Selon elle, le droit de vote féminin est avant tout le sésame de la paix. Contrairement à d'autres féministes, son engagement pacifiste n'est pas une stratégie de reconnaissance politique. D'un pacifisme modéré, avant 1914, elle évolue vers le pacifisme intégral la sclérosant en 1940. C'est aussi une autodidacte auteure de romans, voyageant en Europe pour la paix et le suffrage féminin, soutenant le vote intégral en 1915, favorable au contrôle des naissances, candidate au nom de la parité à la Présidence de la République en 1947, militante de l'abolition du salariat et du partage des richesses.

This research explores the relationship between feminism and pacifism in $\mathrm{XX}^{\text {th }}$-century France through the biography of Jeanne Mélin (1877-1964), pacifist, feminist and writer. The aim is to underline the ambiguity of the angle with which she viewed feminism and pacifism and to explore her understanding of such concepts as feminine identity, masculinity and the difference between the sexes. In her view, women's right to vote constituted an "open sesame" to peace. In opposition to other feminists, her pacifist engagement was not a strategy of political recognition. From a position of moderate pacifism before 1914, she evolved towards an absolute and rigid pacifism in 1940. She was also a self-taught author of novels, travelled widely in Europe on behalf of pacifist and feminist causes, supported universal suffrage in 1915 as well as birth control, and, in 1947, stood as candidate for the Presidency of the Republic in the name of parity of the sexes, campaigning for the abolition of wages and the sharing of all wealth.

\section{INDEX}

Mots-clés : Mélin Jeanne, féminisme, aspect politique, pacifisme, genre

Index géographique : France

\section{AUTEUR}

\section{ISABELLE VAHÉ}

Isabelle VAHÉ est docteure en histoire contemporaine de l'Université Paris 8, elle mène actuellement des recherches sur le pacifisme et le féminisme en France et en Europe dans l'entredeux-guerres. Parmi ses publications : «Jeanne Mélin » (actualisation de la notice de 1976), Dictionnaire biographique du mouvement ouvrier français, 3éme partie : 1870-1914, n²14, Éditions de l'Atelier. Á paraître : « Les relations internationales de la section française de la Ligue 
Internationale des Femmes pour la Paix et la Liberté dans les années 1920 », Sextant, revue du Groupe interdisciplinaire d'Etudes sur les femmes de l'Université Libre de Bruxelles, 2006. 